\title{
OPEN Visualizing the in-vivo application of zinc in sensitive skin using reflectance confocal microscopy
}

\author{
Hye-Jin Ahn ${ }^{1,2}$, Hae Jin Kim², Hyein $\mathrm{Ham}^{3}$, Ji Hwoon Baek ${ }^{3}$, Young Lee ${ }^{4,5}$, Mahin Alamgir ${ }^{5}$, \\ Babar Rao ${ }^{5,6}$ \& Min Kyung Shin ${ }^{1,2,7 凶}$
}

Findings obtained on objective assessments to evaluate sensitive skin do not correlate well with the symptomatology. We utilized reflectance confocal microscopy (RCM) to compare transepidermal application of zinc in sensitive and non-sensitive skin. Thirty-six subjects participated in this study. They were divided into groups based on lactic acid sting test (LAST):'stinger' and 'non-stinger'; transepidermal water loss (TEWL) measurements; and sensitivity self-assessments: 'sensitive' and 'non-sensitive'. RCM images were taken to visualize transepidermal application of topically-applied zinc. The intensity of zinc reflectance at different depths was measured by ImageJ software. Based on LAST scores, the 'stinger' group showed significantly higher reflectance of zinc at $8 \mu \mathrm{m}$ (stratum corneum) [face $(P<0.001)$, forearm $(P=0.004)$ ], and at 80-104 $\mu \mathrm{m}$ (dermo-epidermal junction layer) on the face. High-TEWL group showed increased zinc reflectance at 8-24 $\mu \mathrm{m}$ (tight junction layer, $P<0.001$ ). There were no significant differences amongst subjects self-reporting 'sensitive' and 'nonsensitive' skin. RCM demonstrates that in sensitive skin, there is deeper and higher reflectance of zinc at multiple depths. Structural differences are also visualized. We suggest that RCM is a useful tool for evaluating skin barrier integrity.

There is no widely accepted definition of sensitive skin (SS). However, SS has recently been characterized as a syndrome defined by the occurrence of unpleasant subjective symptoms (stinging, burning, pain, pruritus, and tingling sensations) in response to stimuli that usually should not provoke such sensations $\mathbf{s}^{1,2}$.

Impaired skin barrier function has been suggested to be the underlying cause of $\mathrm{SS}^{3-5}$, and its association with atopic conditions $s^{6-8}$. Several tests have been used to evaluate SS, such as the lactic acid sting test (LAST), thermal sensitivity test, capsaicin test, and histamine test ${ }^{9}$. Objective assessments include measuring the transepidermal water loss (TEWL) after applying sodium lauryl sulfate, dimethylsulfoxide provocation test, colorimetry, corneometry, and laser Doppler velocimetry ${ }^{9}$. However, objective findings do not necessarily correlate with the subjective symptoms ${ }^{9}$. SS is believed to be caused by an increase in the permeability of the stratum corneum (SC), leading to greater transdermal penetration ${ }^{10}$.

Reflectance confocal microscopy (RCM) is a noninvasive method for visualizing the dynamic status of the epidermal and upper dermal structures. In a study using RCM to compare atopic dermatitis and normal skin, the healthy skin showed a uniform arrangement of a polygonal mesh of furrows, which was not seen in the atopic skin $^{11}$. To our knowledge, there have been few studies evaluating the use of RCM to analyze SS in otherwise disease-free skin. We performed in vivo RCM analysis of the skin to evaluate the integrity of the epidermal barrier.

\section{Results}

Zinc reflectance on RCM in groups based on LAST scores. Based on the LAST scores, subjects were divided into a 'stinger' group ( $\mathrm{n}=18$ subjects; 6 males and 12 females), and a 'non-stinger group' ( $\mathrm{n}=18$ subjects; 9 males and 9 females). In the stinger group, zinc application on the face increased progressively from a depth of 0 to $8 \mu \mathrm{m}$ (Fig. 1a). Subsequently, the reflectance decreased and almost leveled off at depths of 24 to $64 \mu \mathrm{m}$.

\footnotetext{
${ }^{1}$ Department of Medicine, Graduate School, Kyung Hee University, Seoul, South Korea. ${ }^{2}$ Department of Dermatology, Kyung Hee University Medical Center, Seoul, South Korea. ${ }^{3}$ Dermapro Skin Research Center, DERMAPRO Ltd, Seoul, South Korea. ${ }^{4}$ Department of Dermatology, School of Medicine, Chungnam National University, Daejeon, South Korea. ${ }^{5}$ Department of Dermatology, Rutgers Robert Wood Johnson Medical School, Somerset, NJ, USA. ${ }^{6}$ Department of Dermatology, Weill Cornell Medical Center, New York, NY, USA. ${ }^{7}$ Department of Dermatology, College of Medicine, Kyung Hee University, \# Kyung HeeDae Ro 23, Dongdaemun-gu, Seoul 02447, South Korea. ${ }^{\circledR}$ email: haddal@hanmail.net
} 
(a)

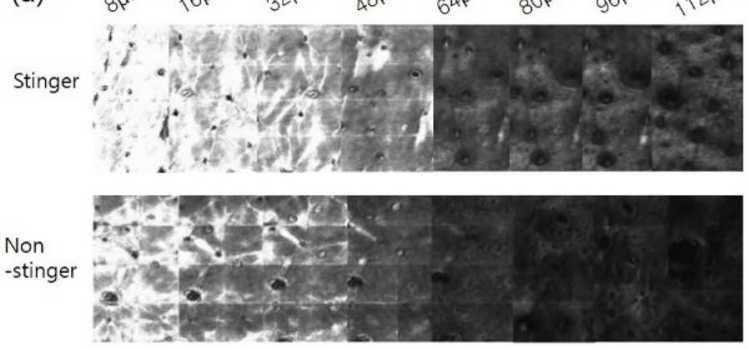

(b)

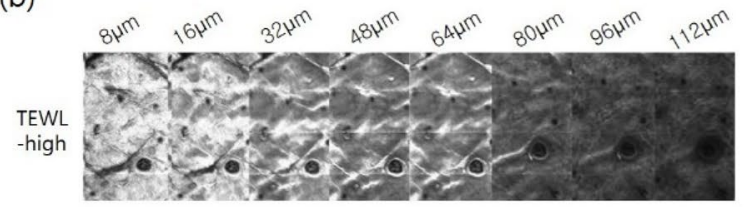

$$
\text { TEWL }
$$

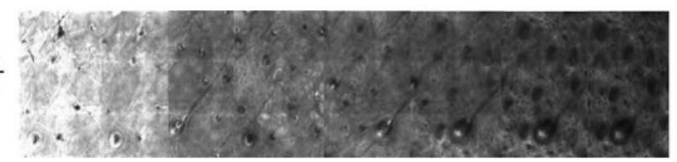

(c)

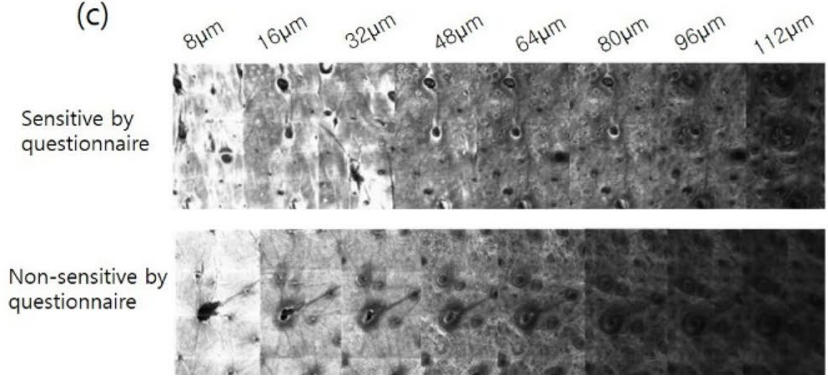

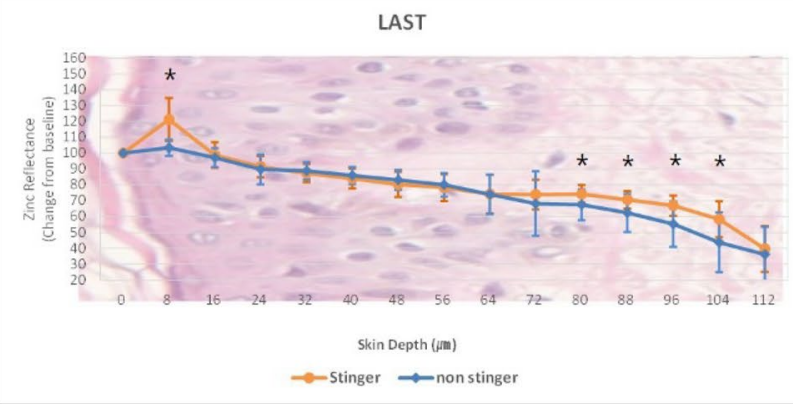

TEWL

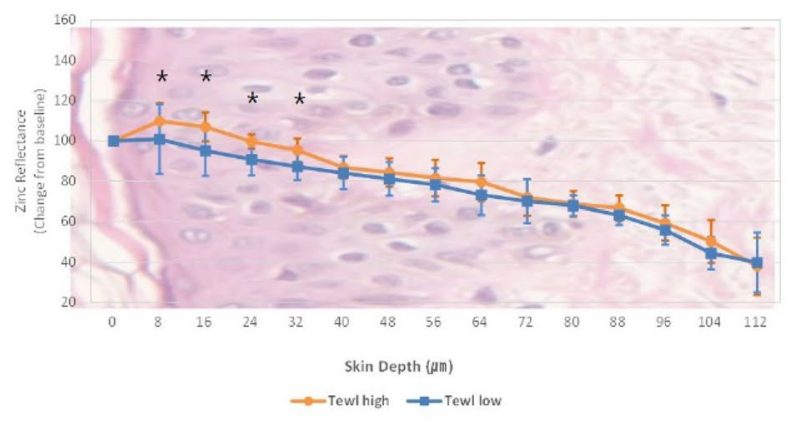

SELF-QUESTIONNAIRE

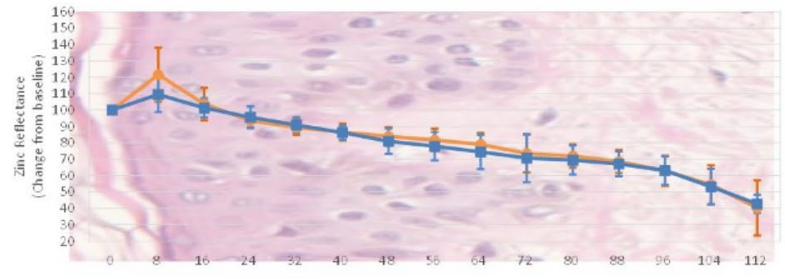

Skin Depth $(\mu m)$

- Sensitive -non Sensitive

Figure 1. RCM images and the zinc reflectance values on the face. The reflectance intensity of zinc at $8 \mu \mathrm{m}$ in the 'stinger' group was higher compared to the non-stinger group $(P<0.001)$. There were significant differences between the two groups from 80 to $104 \mu \mathrm{m}(P=0.048$ at $80 \mu \mathrm{m}, P=0.037$ at $88 \mu \mathrm{m}, P=0.008$ at $96 \mu \mathrm{m}, P=0.042$ at $104 \mu \mathrm{m})(\mathbf{a})$. The high-TEWL group showed greater zinc reflectance from depths of $8 \mu \mathrm{m}$ to $32 \mu \mathrm{m}$ on the face $(P=0.011$ at $8 \mu \mathrm{m}, P<0.001$ at $16-32 \mu \mathrm{m})(\mathbf{b})$. There were no significant differences in zinc reflectance on the face in subjects self-identifying as having 'sensitive' or 'non-sensitive' skin $(\mathbf{c}) .\left({ }^{\star} P<0.05\right)$.

There was a statistically significant difference between the 'stinger' and 'non-stinger' groups at a depth of $8 \mu \mathrm{m}$ and in the deeper layers at depths of 80-104 $\mu \mathrm{m}$ (Supplementary Table S1). Zinc application on the forearm was significantly different only at a depth of $8 \mu \mathrm{m}$ (Fig. 2a, Supplementary Table S1).

RCM images and the zinc reflectance values on the face. The reflectance intensity of zinc at $8 \mu \mathrm{m}$ in the 'stinger' group was higher than that in the non-stinger group $(P<0.001)$. There were significant differences between the two groups from 80 to $104 \mu \mathrm{m}(P=0.048$ at $80 \mu \mathrm{m}, P=0.037$ at $88 \mu \mathrm{m}, P=0.008$ at $96 \mu \mathrm{m}, P=0.042$ at $104 \mu \mathrm{m})(\mathrm{a})$. The high-TEWL group showed greater zinc reflectance from depths of $8 \mu \mathrm{m}$ to $32 \mu \mathrm{m}$ on the face $(P=0.011$ at $8 \mu \mathrm{m}, P<0.001$ at $16-32 \mu \mathrm{m})(\mathrm{b})$. There were no significant differences in zinc reflectance on the face in the subjects who self-assessed themselves as having 'sensitive' or 'non-sensitive' skin (c). $\left({ }^{\star} P<0.05\right)$.

RCM images and the zinc reflectance values on the forearm. The reflectance intensity of zinc at $8 \mu \mathrm{m}$ in the 'stinger' group was greater than that in the 'non-stinger' group $(P=0.004)$ (a). The high-TEWL group showed greater zinc reflectance from 8 to $24 \mu \mathrm{m}$ depth on the forearm $(P=0.009$ at $8 \mu \mathrm{m}, P<0.001$ at $16 \mu \mathrm{m}, P=0.001$ at $24 \mu \mathrm{m})(\mathrm{b})$. There was a significant difference at the $16 \mu \mathrm{m}$ depth $(P=0.003)$ in subjects who self-assessed themselves as having 'sensitive' skin compared to those who self-assessed themselves as having 'non-sensitive' $\operatorname{skin}(\mathrm{c}) .\left({ }^{\star} P<0.05\right)$.

TEWL measurements in the 'stinger' and 'non-stinger' groups. There was no difference in the average TEWL measurements between the 'stinger' and 'non-stinger' groups. The average TEWL on the face was $17.4 \mathrm{gm}^{-2} \mathrm{~h}^{-1}$ in the 'stinger' group and $17.8 \mathrm{gm}^{-2} \mathrm{~h}^{-1}$ in the 'non-stinger' group $(P=0.81)$. The average TEWL on the forearm in the 'stinger' group was $9.3 \mathrm{gm}^{-2} \mathrm{~h}^{-1}$ and it was $8.4 \mathrm{gm}^{-2} \mathrm{~h}^{-1}$ in the 'non-stinger' group $(P=0.37)$ (Supplementary Table S2). 
(a)
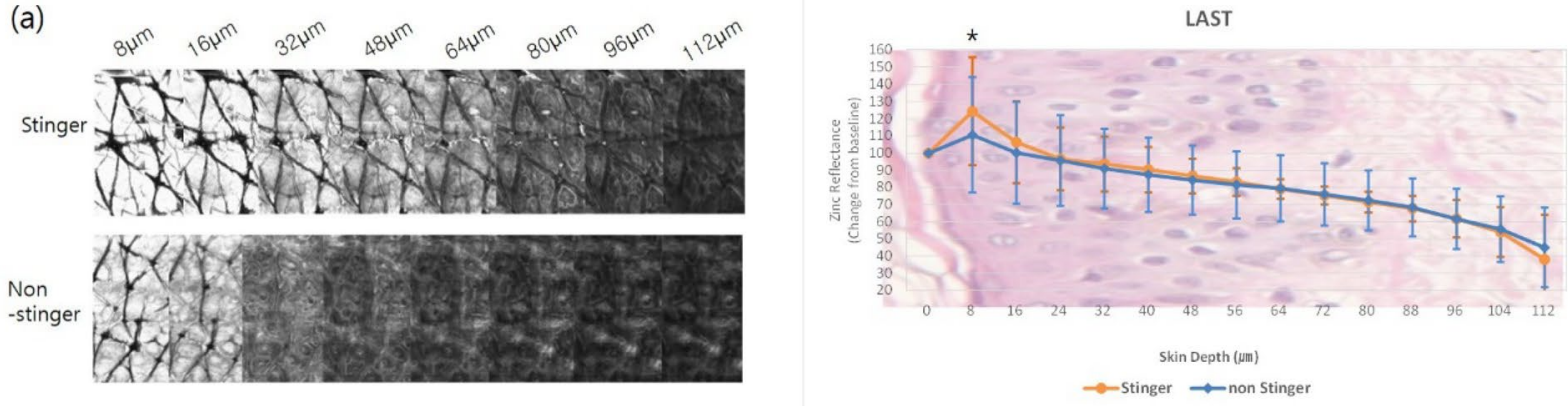

$\rightarrow$ Stinger $\rightarrow$ - non Stinger

TEWL

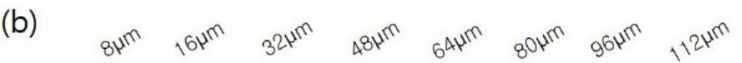
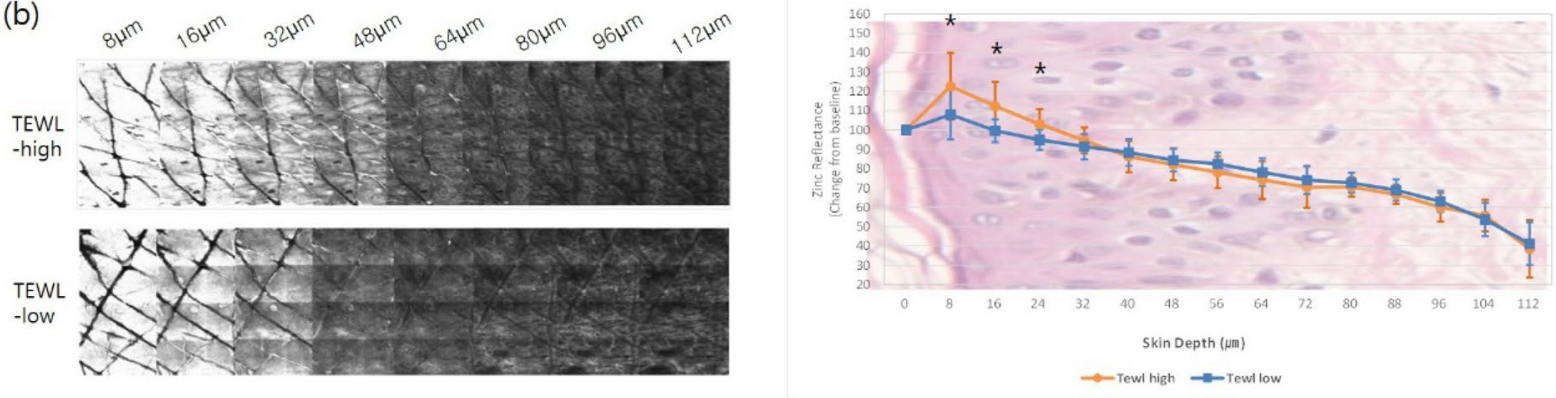

(c) $84^{m}, 64^{m m} \quad 324^{m} \quad 484^{m} \quad 644^{m} \quad 804^{m} \quad 964^{m m}, 124^{m}$
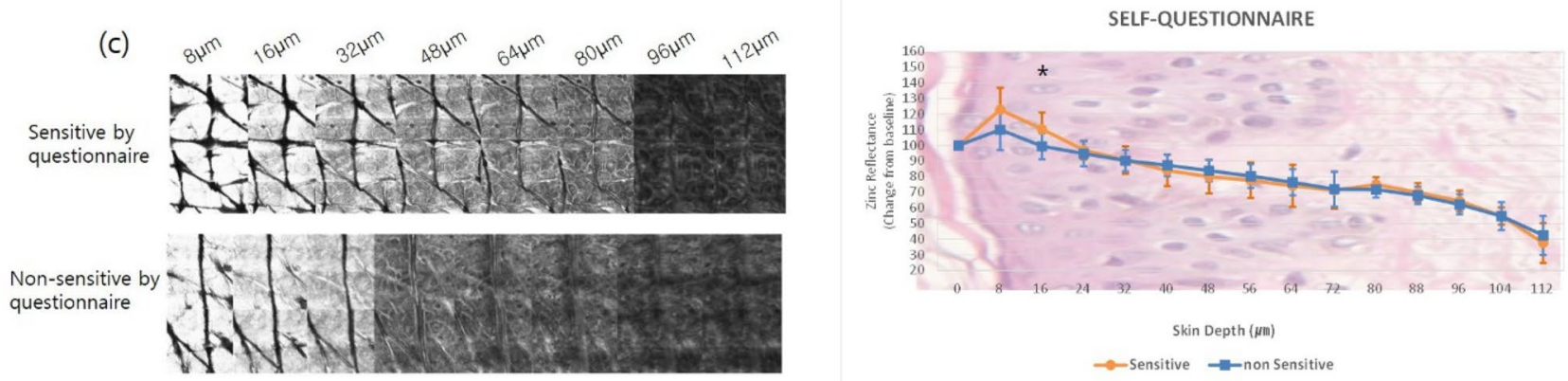

Figure 2. RCM images and the zinc reflectance values on the forearm. The reflectance intensity of zinc at $8 \mu \mathrm{m}$ in the 'stinger' group was greater than the in 'non-stinger' group $(P=0.004)(\mathbf{a})$. The high-TEWL group showed greater zinc reflectance from $8 \mu \mathrm{m}$ to $24 \mu \mathrm{m}$ depth on the forearm $(P=0.009$ at $8 \mu \mathrm{m}, P<0.001$ at $16 \mu \mathrm{m}, P=0.001$ at $24 \mu \mathrm{m})(\mathbf{b})$. There was a significant difference at the $16 \mu \mathrm{m}$ depth $(P=0.003)$ in subjects selfidentifying as having 'sensitive' skin compared to 'non-sensitive' skin $(\mathbf{c}) .\left({ }^{\star} P<0.05\right)$.

Zinc reflectance on RCM in groups based on TEWL measurements. For evaluating the reflectance of zinc on the face, subjects were categorized as high-TEWL $(n=11 ; 8$ males and 3 females) or low-TEWL $(\mathrm{n}=25 ; 7$ males and 18 females). Similarly, for evaluating the reflectance of zinc on the forearm, the subjects were categorized as high-TEWL ( $\mathrm{n}=20,10$ males and 10 females) and low-TEWL ( $\mathrm{n}=16,5$ males and 11 females).

On the face, there were statistically significant differences in the zinc reflectance between the high-TEWL and low-TEWL groups at depths of 8-32 $\mathrm{m}$ (Fig. 1b, Supplementary Table S1). Similarly, on the forearm, RCM showed a significant difference in zinc reflectance at depths of 8 to $24 \mu \mathrm{m}$ (Fig. 2b, Supplementary Table S1).

Zinc reflectance on RCM based on self-assessed skin sensitivity questionnaire. Based on the self-assessment, 26 subjects ( 10 males and 16 females) were grouped into the 'sensitive' group and 10 subjects into the 'non-sensitive' group ( 5 males and 5 females). There were no subjects with co-existing dermatologic disorders. Among the 'sensitive' group, 11 subjects were in the 'stinger' group (based on the results of LAST) and 15 were in the 'non-stinger' group. Among the 'non-sensitive' group, 7 subjects were in the 'stinger' group and 3 subjects were in the 'non-stinger' group. Thus, more subjects made a subjective self-assessment of having SS than the number actually demonstrated through the results of LAST scores.

Among the 'sensitive' group, zinc reflectance on the face was slightly higher than that in the 'non-sensitive' group at depths from 8 to $16 \mu \mathrm{m}$. However, these differences were not significant (Fig. 1c, Supplementary Table S1). On the forearm, higher zinc reflectance was visualized in the 'sensitive' group at depths of 8 to $16 \mu \mathrm{m}$ (Fig. 2c), and this difference was statistically significant at $16 \mu \mathrm{m}$ (Supplementary Table S1).

Dermoscopic images and mosaic RCM images of the groups based on LAST. The mean scores of dermoscopic images in the 'stinger' group were higher (11.53) than in the 'non-stinger' group (10.25) (Sup- 

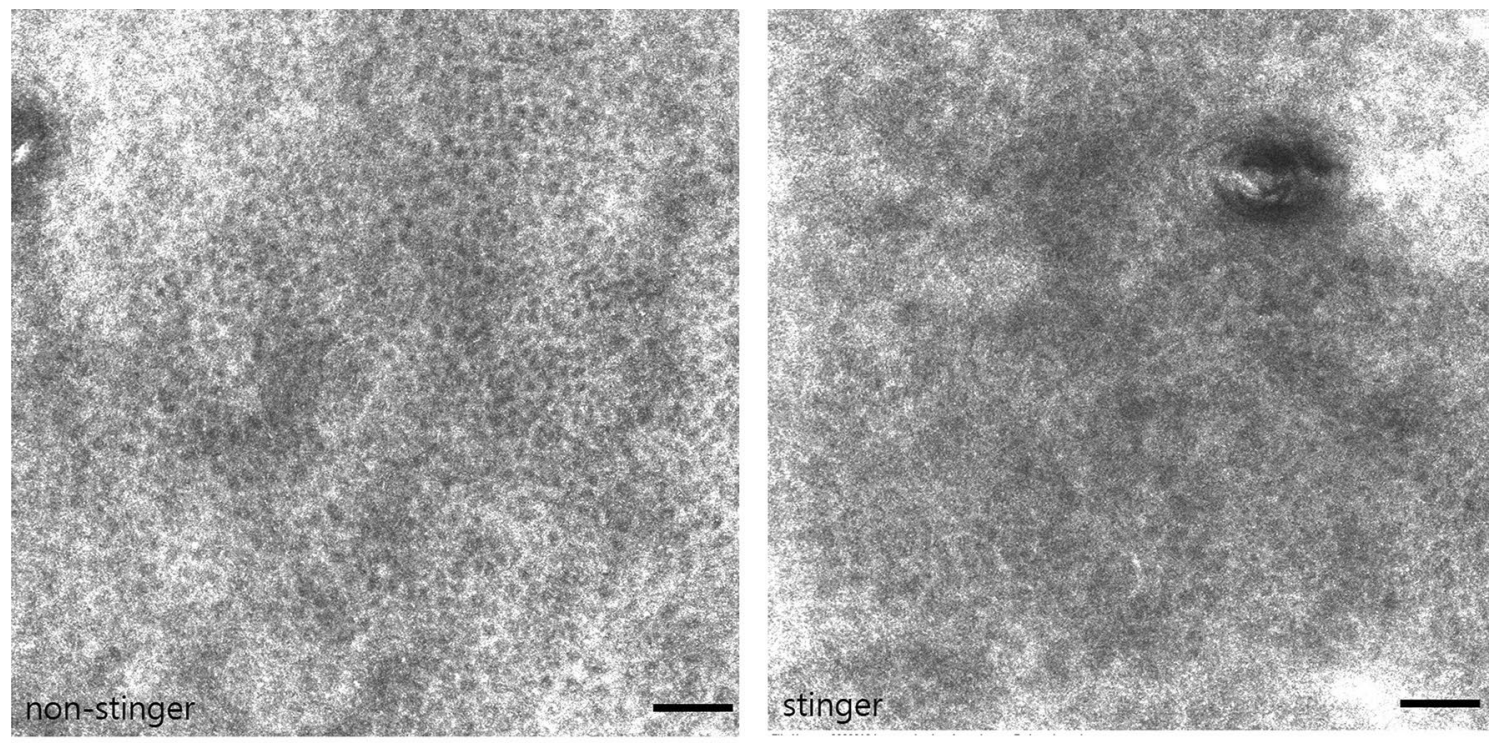

Figure 3. RCM images of the spinous layer on the face. A regular honeycomb pattern in a 'non-stinger' subject and irregular honeycomb pattern in a 'stinger' subject at $24 \mu \mathrm{m}$ depth (Scale bar: $50 \mu \mathrm{m})$.

plementary Figure S3). Among the evaluation criteria, telangiectasia ('stinger': 2.24, 'non-stinger': 1.63) and dyschromia ('stinger': 2.71, 'non-stinger': 2.19) showed the most significant difference between the two groups.

In mosaic RCM images, the mean score of the honeycomb structures in the 'stinger' group (1.06) was slightly higher than that in the 'non-stinger' group (0.81) (Fig. 3). In the images of $500 \times 500 \mu \mathrm{m}^{2}$ on RCM, follicular structures were visualized in 6 subjects in the 'stinger' group and 12 subjects in the 'non-stinger' group. However, there was no apparent perifollicular zinc reflectance in either group.

RCM images of the spinous layer on the face. A regular honeycomb pattern in a 'non-stinger' subject and an irregular honeycomb pattern in a 'stinger' subject at $24 \mu \mathrm{m}$ depth (Scale bar: $50 \mu \mathrm{m}$ ).

\section{Discussion}

The pathophysiology underlying the SS is not completely understood ${ }^{10}$. A systematic study showed that the density of intraepidermal nerve fibers, especially peptidergic C-fibers, is lower in the SS; however, a decrease in epidermal thickness or an increase in the epidermal inflammatory markers was not seen ${ }^{12}$. Previous studies have suggested that the mechanisms underlying SS are neither immunologic nor allergic ${ }^{12-14}$; however, there is an increase in the permeability of the SC, which leads to greater penetration of substances and more water loss ${ }^{10}$.

Several reports have investigated the relationship between the skin barrier and SS. There is an inverse relationship between the corneocyte size/SC thickness and skin permeability, and changes in this mechanical barrier lead to abnormal skin penetration by the irritants ${ }^{15}$. A study reported that in those with SS, the quantity of ceramides in the SC on the face was significantly lower ${ }^{16,17}$, which implies an epidermal barrier dysfunction. However, a recent study investigating the molecular composition of the skin barrier in SS using confocal Raman microspectroscopy found no differences in the SC thickness and the content of water or natural moisturizing factor between the sensitive and non-sensitive skin (NSS) ${ }^{18}$. The authors concluded that the poor correlation between the findings obtained through biophysical techniques and observed alterations in the molecular composition of the skin barrier, emphasizing the need for the use of objective tools for in vivo skin barrier analysis ${ }^{18}$.

In this study, zinc sulfate was used to provide higher reflectivity for RCM images. Zinc, especially zinc oxide nanoparticles that are commonly used in sunscreen formulations. A study of human skin penetration of topical nano zinc oxide showed zinc oxide nonopartiacle failed to penetrate into the viable epidermis of intact human skin, with no detectable levels. In contrast, it was found in the upper layer of the viable epidermis of barrierimpaired skin ${ }^{19}$.

Considering the depth at which a significant decrease in zinc reflectance occurred across all the groups in our study, we suggest that tight junctions, thought to be located in the outermost layer of the stratum granulosum in humans ${ }^{20-22}$, are present at a depth of about $16-24 \mu \mathrm{m}$. Given that in the 'high-TEWL' group, an overall higher zinc reflectance through all the epidermal layers was noted, we postulate that in the skin with high TEWL, concomitant defects in both SC and tight junctions exist. In contrast, the 'stinger' and the self-perceived 'sensitive' group showed only an SC defect, with undamaged tight junctions in the upper epidermal layer. This result is consistent with the increased TEWL seen with defects in tight-junction proteins ${ }^{23}$.

Measurement of TEWL has been used to aid in the diagnosis of $\mathrm{SS}^{10}$. However, a recent study showed that there is no statistical difference between SS and NSS on TEWL measurements ${ }^{5}$. This is in accordance with our findings, which showed no statistical difference in TEWL values between the 'stinger' and 'non-stinger' groups (Supplementary Table S2), despite the 'stinger' group and 'high-TEWL' group showing greater zinc reflectance. Another difference between the 'stinger' group and the 'high-TEWL' group was that the 'stinger' group showed a higher reflectance than the 'non-stinger' group at depths of 80-104 $\mu \mathrm{m}$ on the facial skin. These results suggest 
that the facial skin in the 'stinger' group is associated with an impairment of both the SC barrier and the dermoepidermal junction (DEJ). Using confocal laser scanning microscopy in ex vivo, the bulk of free nerve endings in tissue sections is visualized just below the DEJ ${ }^{24}$. Although a further study is necessary, impairment of the DEJ could be attributed to the sting sensation experienced during LAST. Meanwhile, there are several previous studies that reported epidermal thicknesses measured by RCM. These epidermal thicknesses were $48.28 \mu \mathrm{m}( \pm 13.0)$ in the volar forearm in a younger age group ${ }^{25}$ and $45.07 \mu \mathrm{m}( \pm 10.64)$ in the face in an elderly population ${ }^{26}$. Our result of the DEJ was depths of $80-104 \mu \mathrm{m}$, which was a deeper and wider range compared to the previous studies. Wurm et al. measured the minimal epidermal thickness ${ }^{25}$. Cinotti et al. measured from the top of the $\mathrm{SC}^{26}$. However, we measured depths starting at the base of the SC. Additionally, we suggest the DEJ's wide range appeared to reflect the DEJ's hills-and-valleys topography ${ }^{27}$.

A possible explanation for the difference in reflectance between the face and forearm could be due to regional differences in the size of the corneocyte. Several studies have reported smaller corneocytes in the facial areas relative to those in the forearm ${ }^{28,29}$. The SC of facial skin is thinner and undergoes a more rapid turnover ${ }^{30}$. The unique functional characteristics of the facial skin maintain better hydration of the skin surface, but lead to a relatively weak barrier function ${ }^{30}$.

Some researchers have proposed an association between SS and dermatological diseases such as atopic dermatitis, rosacea, and psoriasis, and several studies using RCM have shown the loss of honeycomb structures and the absence of bright papillary rims in inflammatory skin diseases ${ }^{11,31,32}$. At a depth of $24 \mu \mathrm{m}$, there was an irregular honeycomb pattern in the 'stinger' group on RCM, and we hypothesize that this structural irregularity suggests an existing subclinical inflammatory state.

This is the first study that used RCM to demonstrate in vivo differences in the zinc reflectance of the skin barrier in SS compared to NSS. Our results also demonstrate that the 'stinger' group shows greater reflectance in the SC layer. We further found that findings in the 'high-TEWL' group do not necessarily match those of the 'stinger' group; however, the higher zinc reflectance demonstrated on RCM implies a skin barrier dysfunction in both groups. These differences were not seen among the groups who self-assessed their skin as 'sensitive'. Overall, self-assessments of SS correlated least with the extent of zinc reflectance as visualized on RCM, and thus might be less accurate in predicting actual epidermal barrier defects. In conclusion, RCM demonstrates that in SS, there is a deeper and higher reflectance of zinc at multiple depths. Structural differences were also visualized. We conclude that RCM is a useful tool for evaluating integrity of the skin barrier.

\section{Materials and methods}

Subjects. Thirty-six subjects (15 males, 21 females) aged 20-58 years (mean age, 41.25 years) participated in this study. The DERMAPRO LTD. Institutional Review Board approved the study (220777-A-N-02DICN19040). Informed consent was obtained from all the subjects before enrollment. All the procedures were performed in accordance with relevant guidelines. The subjects were divided into groups based on the LAST scores ('stinger' group $\mathrm{n}=18$, 'non-stinger' group $\mathrm{n}=18$ ), TEWL measurements on the face (high-TEWL group $\mathrm{n}=11$, low-TEWL group $\mathrm{n}=25$ ), TEWL measurements on the forearm (high-TEWL group $\mathrm{n}=20$, low-TEWL group $n=16$ ), and scores of the self-assessed skin sensitivity questionnaire (sensitive group $n=26$, non-sensitive group $n=10)$.

Lactic acid sting test. Each subject's face was washed and stabilized under constant temperature $\left(22 \pm 2{ }^{\circ} \mathrm{C}\right)$ and relative humidity $(50 \pm 5 \%)$ for $30 \mathrm{~min}$. The LAST was conducted according to the Frosch and Kligman method $^{33}$ (" $5 \%$ LAST"); $50 \mathrm{~mL}$ of lactic acid (5\%) and distilled water (DW) were added dropwise to a filter paper $\left(1 \times 1 \mathrm{~cm}^{2}\right)$, which was placed on the nasolabial folds. At $0,2.5,5$, and $8 \mathrm{~min}$, any stinging, burning, or itching reported by the subjects was recorded on a 4-point scale ( 0 , no stinging; 1 , slight stinging; 2 , moderate stinging; 3 , severe stinging). Subjects with scores of 0.2 or higher were selected as a 'stinger' [Score $=$ (Total lactic acid value-Total DW value)/12 (4-point scale $\times 3$ items)].

Transepidermal water loss measurements. The test was conducted in an area away from the wind and direct sunlight. The room temperature was $20-24{ }^{\circ} \mathrm{C}$, and the relative humidity was $45-55 \%$. The probe of a Tewameter TM 300 (Courage \& Khazaka Electronic GmbH, Cologne, Germany) was placed on the face and distal left forearm. The measurement was performed for 30 to $45 \mathrm{~s}$. We defined a high-TEWL on the face as at least 18 gm- 2 h-1 and a high-TEWL on the forearm as at least 8 gm- 2 h- $1^{34}$.

Self-assessed skin sensitivity questionnaire. Participants answered 18 questions about their skin sensitivity and recorded symptoms of skin discomfort, stinging, redness, or dryness following exogenous and endogenous triggers (i.e., toiletries, metals, heat, food, alcohol, emotions). A history of dermatologic disorders such as dermatitis, acne, or rosacea was also obtained. Each question was scored on a scale of 1 to 4 points. A total score of 30 or more was considered as having 'sensitive skin.'

Zinc reflectance on RCM. We captured the real-time scan sequence using RCM (VivaScope1500, Lucid Inc., Rochester, NY) after application of $2 \mathrm{~mL}$ (approximately $1 \mathrm{mg} / \mathrm{cm}^{2}$ ) zinc (zinc sulfate hydrate $4.4 \mathrm{mg} / \mathrm{mL}$; $179.47 \mathrm{~g} / \mathrm{mol}$, Samjin Pharm, South Korea) on the face and forearm. RCM images were taken $60 \mathrm{~min}$ after topical application. Zinc provided high reflectance on the RCM images (Fig. 4). We used laser power at 5.8-6.0 W and horizontal composite images of $4 \times 4 \mathrm{~mm}^{2}$ (confocal mosaics) for the analysis. We used ImageJ software to measure the intensity of zinc reflectance. Since the reflectance of RCM according to the melanin content varies between each individual, we corrected the zinc reflectance by subtracting the baseline reflectance value of each skin layer. Baseline reflectance was obtained from the surrounding skin without zinc application. We considered 

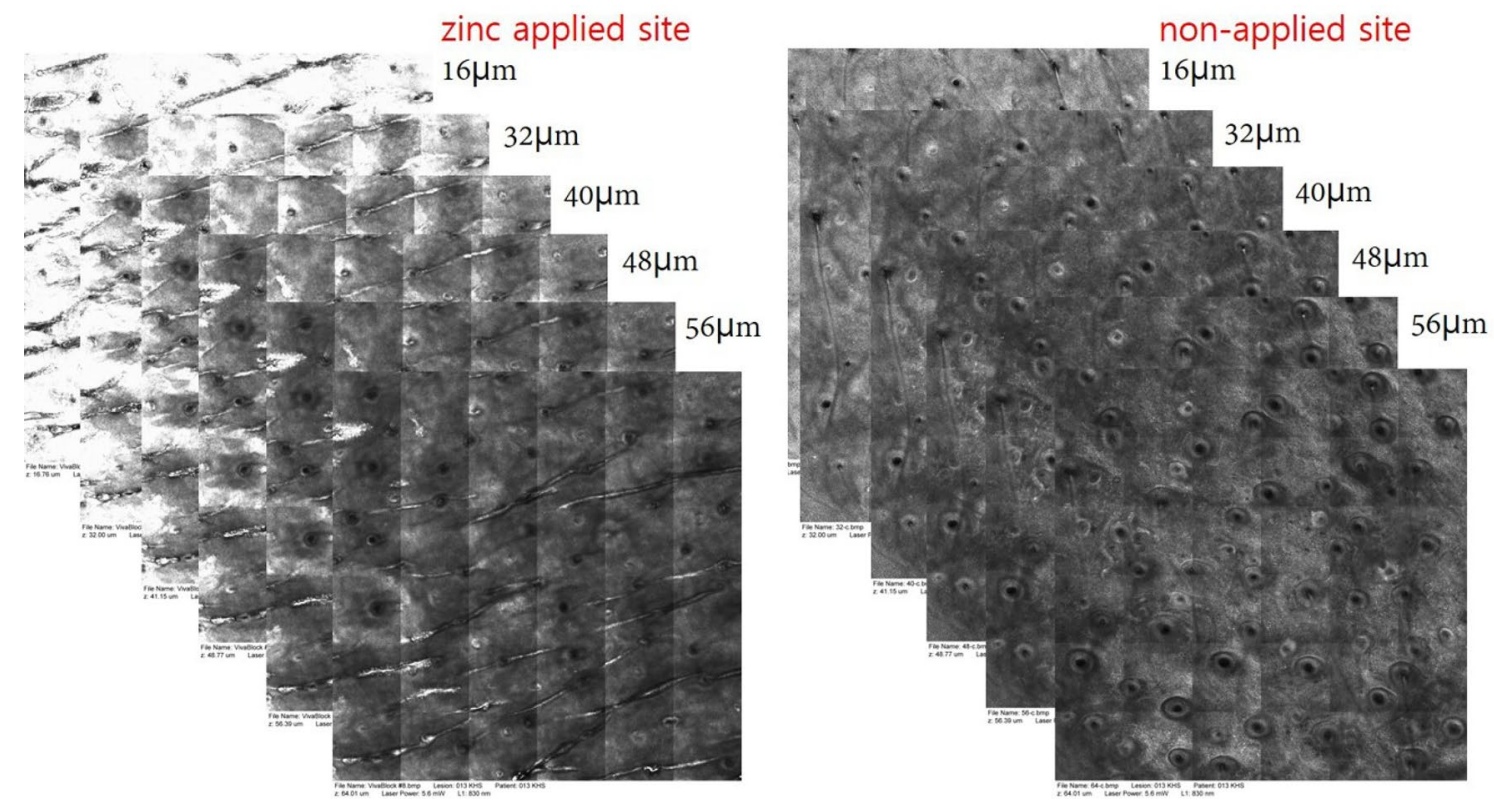

Figure 4. RCM images after zinc application. After zinc application, the facial skin showed higher reflectance than the non-applied zinc facial skin at different depths.

the deep layer of the SC as the most superficial epidermal layer $(0 \mu \mathrm{m})$. We set the zinc reflectance intensity value as 100 at an epidermal depth of $0 \mu \mathrm{m}$, and the remaining values were calculated as relative values. Finally, the corrected intensity values at each skin depth were plotted on graphs.

RCM images after zinc application. After zinc application, the facial skin showed higher reflectance at different depths than that of the areas on which zinc was not applied.

Analysis of dermoscopic and mosaic images of the groups based on LAST. We analyzed the dermoscopic images taken from 'stinger' and 'non-stinger' groups (based on the LAST scores) using a digital macro camera (Vivacam; Lucid Inc., Rochester, NY), which was linked to the RCM. Dermoscopic images were evaluated for erythema, telangiectasias, eccrine duct orifice hyperpigmentation, perifollicular hyperkeratosis, and dyschromia. Scores were assigned to each feature (1: none, 2: weak, 3: strong). We also captured the RCM images showing the honeycomb structures and perifollicular zinc reflectance at a depth of $24 \mu \mathrm{m}$ in the 'stinger' and 'non-stinger' groups. The quality of the honeycomb structure was determined as: 0, regular structure; 1 , slightly irregular; and 2, very irregular.

Statistical analysis. We performed a Wilcoxon rank-sum test to identify significant differences in the zinc application reflectance, and a t-test was used to compare the TEWL between the 'stinger' and 'non-stinger' groups (based on LAST scores). Data were analyzed using SPSS for Windows (version 23.0, SPSS Inc., Chicago, IL). For all statistical tests, a P-value $<0.05$ was considered statistically significant.

Received: 21 October 2020; Accepted: 17 March 2021

Published online: 08 April 2021

\section{References}

1. Del Rosso, J., Zeichner, J., Alexis, A., Cohen, D., Berson, D. Understanding the epidermal barrier in healthy and compromised skin: clinically relevant information for the dermatology practitioner Proceedings of an Expert Panel Roundtable Meeting. J. Clin. Aesthet Dermatol. 9(4), S2-S8 (2016).

2. Misery, L. et al. Definition of sensitive skin: an expert position paper from the special interest group on sensitive skin of the International Forum for the Study of Itch. Acta Derm. Venereol. 97, 4-6 (2017).

3. Distante, F., Rigano, L., D’Agostino, R., Bonfigli, A. \& Berardesca, E. Intra- and inter-individual differences in sensitive skin. Cosmet. Toiletries. 117, 39-46 (2002).

4. An, S. et al. Comparison and correlation between stinging responses to lactic acid and bioengineering parameters. Contact Dermatitis 57, 158-162 (2007).

5. Richters, R. J. et al. Clinical, biophysical and immunohistochemical analysis of skin reactions to acute skin barrier disruption: a comparative trial between subjects with sensitive skin and subjects with non-sensitive skin. Br. J. Dermatol. 174, 1126-1133 (2016).

6. De Jongh, C. M. et al. Stratum corneum cytokines and skin irritation response to sodium lauryl sulfate. Contact Dermatitis 54, 325-333 (2006).

7. Farage, M. A., Katsarou, A. \& Maibach, H. I. Sensory, clinical and physiological factors in sensitive skin: a review. Contact Dermatitis 55, 1-14 (2006).

8. Willis, C. M. et al. Sensitive skin: an epidemiological study. Br. J. Dermatol. 145, 258-263 (2001). 
9. Kim, Y. R., Cheon, H. I., Misery, L., Taieb, C. \& Lee, Y. W. Sensitive skin in Korean population: an epidemiological approach. Skin Res. Technol. 24, 229-234 (2018).

10. Duarte, I., Jéssica Eleonora, P., Silveira, S., de Figueiredo Silva Hafner, M., Toyota, R., Debora Midori, Pedroso, M. Sensitive skin. Review of an ascending concept. An. Bras. Dermatol. 92, 521-525 (2017).

11. Meinke, M. C. et al. Characterization of atopic skin and the effect of a hyperforin-rich cream by laser scanning microscopy. J. Biomed. Opt. 20(5), 051013 (2015).

12. Buhé, V. et al. Pathophysiological study of sensitive skin. Acta Derm. Venereol. 96, 314-318 (2016).

13. Misery, L. Sensitive skin. Expert Rev. Dermatol. 8, 631-637 (2013).

14. Ständer, S., Schneider, S. W., Weishaupt, C., Luger, T. A. \& Misery, L. Putative neuronal mechanisms of sensitive skin. Exp. Dermatol. 18, 417-423 (2009).

15. Beradesca, E. Tests for sensitive skin. In: Borel, A.O., Paye, M., Maibach, H.I., editors. Handbook of Cosmetic Science and Technology. 4th ed. Boca Raton: CRC Press/Taylor \& Francis Group, cop, 77-79 (2014)

16. di Nardo, A., Sugino, K., Wertz, P., Ademola, J. \& Maibach, H. I. Sodium lauryl sulfate (SLS) induced irritant contact dermatitis: a correlation study between ceramides and in vivo parameters of irritation. Contact Dermatitis 35, 86-91 (1996).

17. Cho, H. J. et al. Quantitative study of stratum corneum ceramides contents in patients with sensitive skin. J. Dermatol. 39, 295-300 (2012).

18. Richters, R. J. et al. Sensitive Skin: assessment of the skin barrier using confocal Raman microspectroscopy. Skin Pharmacol. Physiol. 30, 1-12 (2017).

19. Leite-Silva, V. R. et al. Human skin penetration and local effects of topical nano zinc oxide after occlusion and barrier impairment. Eur. J. Pharm. Biopharm. 104, 140-147 (2016).

20. Ishida-Yamamoto, A. et al. Lamellar granule secretion starts before the establishment of tight junction barrier for paracellular tracers in mammalian epidermis. PLoS ONE 7, e31641 (2012).

21. Ulrich, M., Lange-Asschenfeldt, S. \& Gonzalez, S. The use of reflectance confocal microscopy for monitoring response to therapy of skin malignancies. Dermatol. Pract. Concept. 2(2), 0202a10 (2012).

22. Yokouchi, M. \& Kubo, A. Maintenance of tight junction barrier integrity in cell turnover and skin diseases. Exp. Dermatol. 27, 876-883 (2018).

23. Turksen, K. \& Troy, T.-C. Permeability barrier dysfunction in transgenic mice overexpressing claudin 6. Development 129, 1775$1784(2002)$.

24. Tschachler, E. et al. Sheet preparations expose the dermal nerve plexus of human skin and render the dermal nerve end organ accessible to extensive analysis. J. Invest. Dermatol. 122(1), 177-182 (2004).

25. Wurm, E. M. et al. In vivo assessment of chronological ageing and photoageing in forearm skin using reflectance confocal microscopy. Br. J. Dermatol. 167, 270-279 (2012).

26. Cinotti, E. et al. Structural skin changes in elderly people investigated by reflectance confocal microscopy. J. Eur. Acad. Dermatol. Venereol. 34, 2652-2658 (2020).

27. Kurugol, S., Dy, J. G., Brooks, D. H. \& Rajadhyaksha, M. Pilot study of semiautomated localization of the dermal/epidermal junction in reflectance confocal microscopy images of skin. J. Biomed. Opt. 16, 036005 (2011).

28. Plewig, G. \& Marples, R. R. Regional differences of cell sizes in the human stratum corneum. Part I. J. Invest. Dermatol. 54, 13-18 (1970).

29. Rougier, A., Lotte, C., Corcuff, P. \& Maibach, H. Relationship between skin permeability and corneocyte size according to anatomic site, age and sex in man. J. Soc. Cosmet. Chem. 39, 15-26 (1988).

30. Tagami, H. Location-related differences in structure and function of the stratum corneum with special emphasis on those of the facial skin. Int. J. Cosmet. Sci. 30, 413-434 (2008).

31. Slodownik, D., Williams, J., Lee, A., Tate, B. \& Nixon, R. Controversies regarding the sensitive skin syndrome. Expert Rev. Dermatol. 2, 579-584 (2007).

32. Mills, O. H. \& Berger, R. S. Defining the susceptibility of acne-prone and sensitive skin population to extrinsic factors. Dermatol. Clin. 1, 93-98 (1991).

33. Frosch, P. J. \& Kligman, A. M. A method for appraising the stinging capacity of topically applied substances. J. Soc. Cosmet. Chem. 28, 197-209 (1977).

34. Akdeniz, M., Gabriel, S., Lichterfeld-Kottner, A., Blume-Peytavi, U. \& Kottner, J. Transepidermal water loss in healthy adults: a systematic review and meta-analysis update. Br. J. Dermatol. 179, 1049-1055 (2018).

\section{Acknowledgements}

This work was supported by a grant from Korean Research Foundation in 2017 (NRF-2017R1C1B5076162).

\section{Author contributions}

M.K.S. designed the study. H.J.K., H.H. and J.H.B. conducted the LAST, TEWL and RCM measurement. H.J.A. and M.K.S. analyzed the data. H.-J.A. wrote the manuscript. Y.L., M.A. and B.R. helped in revising the manuscript.

\section{Competing interests}

The authors declare no competing interests.

\section{Additional information}

Supplementary Information The online version contains supplementary material available at https://doi.org/ 10.1038/s41598-021-87346-0.

Correspondence and requests for materials should be addressed to M.K.S.

Reprints and permissions information is available at www.nature.com/reprints.

Publisher's note Springer Nature remains neutral with regard to jurisdictional claims in published maps and institutional affiliations. 
(c) (i) Open Access This article is licensed under a Creative Commons Attribution 4.0 International cc) License, which permits use, sharing, adaptation, distribution and reproduction in any medium or format, as long as you give appropriate credit to the original author(s) and the source, provide a link to the Creative Commons licence, and indicate if changes were made. The images or other third party material in this article are included in the article's Creative Commons licence, unless indicated otherwise in a credit line to the material. If material is not included in the article's Creative Commons licence and your intended use is not permitted by statutory regulation or exceeds the permitted use, you will need to obtain permission directly from the copyright holder. To view a copy of this licence, visit http://creativecommons.org/licenses/by/4.0/.

(C) The Author(s) 2021 